\title{
ESTABILIDADE QUÍMICA E MICROBIOLÓGICA DE "MINCED FISH" DE PEIXES AMAZÔNICOS DURANTE O CONGELAMENTO1
}

\author{
Rogério Souza de JESUS², Edson LESSI², Alfredo TENUTA-FILHO ${ }^{3, *}$
}

\begin{abstract}
RESUMO
Foi estudada a estabilidade química e microbiológica de "minced fish" produzidos, em condições industriais, com espécies de peixes da Amazônia: aracú-comum (Schizodon fasciatus), branquinhacomum (Potamorhina latior), branquinha-de-cabeça-lisa $(P$. altamazonica), curimatã (Prochilodus nigricans), jaraqui-de-escama-fina (Semaprochilodus taeniurus), jaraqui-de-escama-grossa (S. insignis), mapará (Hypophthalmus edentatus), pacú-comum (Metynnis hypsauchen), pacú-manteiga (Mylossoma duriventre) e pirapitinga (Piaractus brachypomum), durante 150 dias sob congelamento a $-18 \pm 1^{\circ} \mathrm{C} \mathrm{e}-36 \pm 1^{\circ} \mathrm{C}$. Com base no $\mathrm{pH}$, nitrogênio das bases voláteis totais (N-BVT), substâncias reagentes ao ácido tiobarbitúrico (TBARS) e contagens de aeróbios mesófilos a $35^{\circ} \mathrm{C}$ e psicrotróficos a $7^{\circ} \mathrm{C}$, e de coliformes totais e fecais (NMP), os "minced fish" obtidos a partir das espécies de peixes estudadas e de misturas de espécies (aracú+curimatã+pirapitinga; jaraqui+branquinha) mantiveram-se em condições de consumo, durante o período experimental. Os resultados obtidos são altamente promissores sob o ponto de vista tecnológico.
\end{abstract}

Palavras-chave: peixe de água-doce; peixe amazônico "minced fish"; qualidade química; qualidade microbiológica; congelamento.

\section{SUMMARY}

CHEMICAL AND MICROBIOLOGICAL STABILITY OF AMAZONIAN MINCED FISH DURING THE FREEZING. This work intended to study the stability of some minced freshwater fish species, available in the Amazon region: aracú-comum (Schizodon fasciatus), branquinha-comum (Potamorhina latior), branquinha-de-cabeça-lisa ( $P$. altamazonica), curimatã (Prochilodus nigricans), jaraqui-de-escama-fina (Semaprochilodus taeniurus), jaraqui-de-escama-grossa ( $S$. insignis), mapará (Hypophthalmus edentatus), pacú-comum (Metynnis hypsauchen), pacú-manteiga (Mylossoma duriventre) and pirapitinga (Piaractus brachypomum), produced under industrial conditions and frozen stored. During a 150 day period, chemical and microbiological stability of the minced fish, under frozen temperatures of $-18 \pm 1^{\circ} \mathrm{C}$ and $-36 \pm 1^{\circ} \mathrm{C}$, were monthly determined by evaluation of $\mathrm{pH}$, nitrogen of total volatile bases (N-BVT), thiobarbituric acid reactant substances (TBARS) and mesophile and psycrotrophic aerobic counts at $35^{\circ} \mathrm{C}$ and $7^{\circ} \mathrm{C}$, respectively, and total and fecal coliforms (MPN). The results were compared, and we could conclude that the different species under study have important technological characteristics. Minced fish prepared with the seven species under study were fit for consumption, from a chemical and microbiological point of view, during the 150 -day period of storage at -18 and $-36^{\circ} \mathrm{C}$.

${ }^{1}$ Recebido para publicação em 29/06/99. Aceito para publicação em 11/07/01.

${ }^{2}$ Coordenação de Pesquisas em Tecnologia de Alimentos, INPA, Av. André Araújo, 1756, Aleixo, CEP 69083-000 MANAUS (AM)E-mail: djesus@inpa.gov.br

${ }^{3}$ Departamento de Alimentos e Nutrição Experimental, Faculdade de Ciências Farmacêuticas, USP, Av. Prof. Lineu Prestes, 580, Bloco 14. Cid. Universitária, CEP 05508-900 SÃO PAULO (SP) E-mail: eetenuta@usp.br

${ }^{*}$ A quem a correspondência deve ser enviada.
Keywords: freshwater fish; amazonian fish; minced fish; chemical quality; microbiological quality; freezing.

\section{1 - INTRODUÇÃO}

A mudança nos hábitos sociais e o aumento do poder aquisitivo dos consumidores têm permitido o consumo de pescado, que anteriormente era consumido in natura, já parcialmente ou inteiramente elaborado. Entre esses novos tipos de produtos de pescado, estão aqueles que utilizam o "minced fish" e/ou o "surimi" como seu principal ingrediente.

Os produtos de "minced fish" são de uma geração mais antiga que aqueles à base de "surimi", e normalmente são vistos como tendo seu lugar ocupado cada vez mais por estes últimos, no entanto o seu processo de produção é relativamente simples e mais amplamente aplicável. O "minced fish" é definido pelo Codex Alimentarius como produto obtido a partir de uma única espécie ou mistura de espécies de peixes com características sensoriais similares, submetido a processo de separação mecânica, resultando em partículas de músculo esquelético isentas de ossos, vísceras e pele [2]. Na realidade, é possível a utilização de elementos tecnológicos do processamento do "surimi" para a melhoria do "minced fish" e seus produtos; os quais apresentam vantagens quanto ao aumento na utilização da matéria-prima e diminuição de perdas no processamento.

Cerca de 60.000 toneladas de peixes são desembarcadas em Manaus (AM) anualmente. Essa produção tem sido usada para o consumo local e também em outras regiões, no país e exterior. Entre cerca de 2000 espécies de peixes existentes nos rios amazônicos, aparecem no mercado de Manaus em torno de 40 espécies, das quais somente 8 a 10 possuem expressão econômica. Os produtos de pescado amazônicos exportados, ou são congelados inteiros ou em forma de filés, concentrando-se nas espécies de bagres [3].

Potencialmente a produção do "minced fish" elaborado a partir de espécies de peixes amazônicos com baixo valor comercial, pode representar uma opção tecnológica economicamente viável para a região. Em consequência haveria a desconcentração do esforço-de-pesca sobre as espécies mais preferidas, aumento do aproveitamento do músculo do pescado em função do maior rendimento alcançado por essa tecnologia, e a possibilidade de instalação de pequenas e médias empresas na região, aumentando a oferta de novos produtos de pescado e o emprego da mãode-obra local. 
Neste trabalho, nove tipos de "minced fish" elaborados com espécies ou misturas de espécies de peixes amazônicos foram avaliados química e microbiologicamente, durante o armazenamento congelado a $-18^{\circ} \mathrm{C}$ e $-36^{\circ} \mathrm{C}$. As espécies foram selecionadas com base na sua abundância e baixo valor comercial.

\section{2 - MATERIAL E MÉTODOS}

\section{1 - Material}

Foram empregadas as espécies: aracú-comum (Schizodon fasciatum), branquinha (mistura indeterminada de branquinha-comum, Potamorhina latior e branquinha-de-cabeça-lisa, $P$. altamazonica), curimatã (Prochilodus nigricans), jaraqui (mistura indeterminada de jaraqui-de-escama-grossa, Semaprochilodus insignis, e jaraqui-de-escama-fina, S. taeniurus), mapará (Hypophthalmus edentatus), pacú (mistura indeterminada de pacú-comum, Metynnis hypsauchen e pacúmanteiga, Mylossoma duriventre) e pirapitinga (Piaractus brachypomum), capturadas em rios da Amazônia e desembarcadas no Porto de Desembarque de Pescado, de Manaus (AM). Adquiridas diretamente junto às embarcações pesqueiras, as amostras foram mantidas em gelo triturado e transportadas para uma indústria de pesca em Iranduba (AM), para serem processadas.

\section{2 - Obtenção dos "minced fish"}

Os peixes foram decapitados, eviscerados, lavados com água clorada (5mg/L de $\mathrm{Cl}^{-}$livre), cortados longitudinalmente e submetidos ao processamento de separação mecânica do músculo, em separadora alemã Baader modelo 694. Por apresentar alta concentração lipídica subcutânea, as amostras de mapará foram previamente filetadas, para evitar excesso de lípides e resíduos indesejáveis de pele no produto final. Os "minced fish" obtidos foram distribuídos em bandejas metálicas, na forma de blocos, e congelados imediatamente em congelador de placas a $-30^{\circ} \mathrm{C}$.

Os blocos congelados, originalmente com cerca de $7 \mathrm{~kg}$ de peso e espessura de $5 \mathrm{~cm}$ cada um, foram retaIhados com serra-fita elétrica, em porções com cerca de $500 \mathrm{~g}$, e embalados em filme de polietileno. Foram preparados "minced fish" individuais, a partir das espécies: aracú-comum, branquinha, curimatã, jaraqui, mapará, pacú e pirapitinga, e de misturas das espécies: aracú, curimatã e pirapitinga (1:1:1) e branquinha e jaraqui (1:1).

Os blocos congelados de "minced fish", acondicionados em caixas isotérmicas contendo gelo seco, foram transportados (via aérea) para o Laboratório de Química e Bioquímica de Produtos Animais, do Departamento de Alimentos e Nutrição Experimental da Faculdade de Ciências Farmacêuticas/USP, em São Paulo (SP), e estocados a $-18 \pm 1^{\circ} \mathrm{C}$ e $-36 \pm 1^{\circ} \mathrm{C}$.

\section{3 - Avaliação da estabilidade química e microbiológica}

Mensalmente, durante 150 dias de estocagem a $-18 \pm 1^{\circ} \mathrm{C}$ e $-36 \pm 1^{\circ} \mathrm{C}$, as amostras de "minced fish" foram descongeladas à temperatura ambiente, por cerca de três horas, e submetidas às análises correspondentes.

$\mathrm{O} \mathrm{pH}$ foi determinado segundo as Normas Analíticas do Instituto Adolfo Lutz [12] e o nitrogênio das bases voláteis totais (N-BVT) pela adaptação do método descrito por WOOTLON \& CHUAH [13]. As substâncias reativas ao ácido tiobarbitúrico (TBARS) foram determinadas de acordo com o método descrito por WYNCKE [14], modificado quanto ao tempo de reação entre o extrato e o reativo, sob temperatura de ebulição, que foi reduzido de 40 para 10 minutos. As análises microbiológicas constaram de contagens de aeróbios mesófilos a $35^{\circ} \mathrm{C}$, psicrotróficos a $7^{\circ} \mathrm{C}$ e de coliformes totais e fecais (NMP), efetuadas em amostras armazenadas durante 120 dias a $-18^{\circ} \mathrm{C}$. Para isso, as amostras foram descongeladas em refrigerador, durante 18 horas, antes das análises [1].

\section{4 - Análises estatísticas}

A comparação de médias foi feita pelos testes tstudent ou Mann-Whitney. Foi estabelecida a função ajustada significativa das variáveis analisadas com o tempo, pelo teste de correlação de Pearson [15]. A significância de $95 \%$ foi adotada para a análise dos resultados.

\section{3 - RESULTADOS E DISCUSSÃO}

Os valores de $\mathrm{pH}$ dos diferentes "minced fish" estocados $\mathrm{a}-18 \pm 1^{\circ} \mathrm{C}$ e $-36 \pm 1^{\circ} \mathrm{C}$ mostraram pouca variação ao longo dos 150 dias de estocagem, ficando entre 6,50 e 7,07. Os "minced fish" de mapará e pacú apresentaram valores de $\mathrm{pH}$ superiores a 6,75 no início do experimento. $\mathrm{Na}$ temperatura de $-36^{\circ} \mathrm{C}$, todas as amostras apresentaram aumento do valor de $\mathrm{pH}$ ao longo do tempo. $\mathrm{A}-18^{\circ} \mathrm{C}$, os "minced fish" de pirapitinga e A+C+P (aracú, curimatã e pirapitinga) não apresentaram correlação positiva com o tempo de estocagem, ao contrário do que ocorreu com as demais amostras (Tabela 1). Os valores de $\mathrm{pH}$ dos "minced fish" estocados a $-18^{\circ} \mathrm{C}$ e $-36^{\circ} \mathrm{C}$ não apresentaram diferença significante $(p>0,05)$ entre as duas temperaturas, com exceção para o "minced fish" $A+C+P(p=0,027)$.

RODRIGUEZ \& BELLO [10] trabalharam com blocos congelados de "minced fish" de peixes da fauna acompanhante da pesca de camarão, estocados a $-10^{\circ} \mathrm{C}$ e $-30^{\circ} \mathrm{C}$, durante 6 meses. Os autores verificaram que não ocorreu alteração significativa de $\mathrm{pH}$ em ambas temperaturas, ainda que a $-10^{\circ} \mathrm{C}$ tenha ocorrido acúmulo de bases. GUTIÉRREZ \& BELLO [4] também não encontraram mudanças de $\mathrm{pH}$ significantes ao longo do tempo, sob temperatura de $-20^{\circ} \mathrm{C}$, quando estudaram produtos elaborados de "minced fish" com sabor de camarão.

Segundo LOVE [9], o pH do músculo do pescado é de grande importância tecnológica, por ser o principal fator relacionado com a textura do músculo cozido. $A$ importância do $\mathrm{pH}$ do músculo do peixe se reflete principalmente na textura e no "gaping", fenômeno de ruptura que ocorre nos filés congelados, prejudicando sua 
qualidade para comercialização ou o processamento em produtos integrais. No "minced fish", certamente esse problema teria menor relevância, devido ao processo de separação mecânica que desintegra o produto.

Os valores de nitrogênio das bases voláteis totais (N-BVT) dos diferentes "minced fish" estocados a $-18 \pm 1^{\circ} \mathrm{C}$ e $-36 \pm 1^{\circ} \mathrm{C}$ mostraram tendência decrescente ao longo do tempo de estocagem, embora essa queda não tenha sido significante estatisticamente. Os limites máximo e mínimo ficaram entre $23,3 \mathrm{mgN} / 100 \mathrm{~g}$, no "minced fish" da mistura de $\mathrm{A}+\mathrm{C}+\mathrm{P}$, no início do experimento, e $13,7 \mathrm{mgN} / 100 \mathrm{~g}$, para os de mapará e pacú, ao final do período de armazenagem (Tabela 2). A comparação das médias dos valores de N-BVT entre os "minced fish", com relação às temperaturas de $-18^{\circ} \mathrm{C}$ e $-36^{\circ} \mathrm{C}$, não apresentou diferença significante $(p>0.05)$. O valor máximo observado nos "minced fish" situou-se abaixo de $30,0 \mathrm{mgN}-B V T / 100 \mathrm{~g}$, limite estabelecido pela legislação para o pescado e produtos derivados, para efeito de consumo [11].

RODRIGUEZ \& BELLO [10], LANDA \& MARTINEZ [8] e GUTIÉRREZ \& BELLO [4] demonstraram a ocorrência de aumento dos teores de N-BVT durante o congelamento de "minced fish" e seus produtos, diferentemente do observado no presente trabalho (Tabela 2). O N-BVT tem sido utilizado para estimar objetivamente a qualidade do pescado, onde é de se esperar que à medida que as contagens microbianas sejam mais elevadas, seus valores aumentem. Os resultados da Tabela 2 podem portanto ser interpretados como uma redução da atividade microbiana durante o congelamento, fato sugerido pela queda nas contagens de aeróbios mesófilos e psicrotróficos, conforme indicado na Tabela 4.

TABELA 1. Valores de pH em "minced fish" estocados sob congelamento durante 150 dias

\begin{tabular}{|c|c|c|c|c|c|c|c|}
\hline \multirow[t]{2}{*}{ Espécies } & \multirow{2}{*}{$\begin{array}{l}\text { Temperatura } \\
\text { de estocagem }\end{array}$} & \multirow[t]{2}{*}{ Início } & \multicolumn{5}{|c|}{ TEMPO DE ESTOCAGEM ( dias) } \\
\hline & & & 30 & 60 & 90 & 120 & 150 \\
\hline \multirow[t]{2}{*}{ Aracú-comum } & $-18^{\circ} \mathrm{C}$ & 6,67 & 6,85 & 6,81 & 6,92 & 6,84 & 6,85 \\
\hline & $-36^{\circ} \mathrm{C}$ & 6,67 & 6,99 & 6,73 & 6,98 & 6,95 & 7,04 \\
\hline \multirow[t]{2}{*}{ Branquinha } & $-18^{\circ} \mathrm{C}$ & 6,61 & 6,78 & 6,78 & 6,81 & 6,75 & 6,81 \\
\hline & $-36^{\circ} \mathrm{C}$ & 6,61 & 6,87 & 6,67 & 6,92 & 6,82 & 6,94 \\
\hline \multirow[t]{2}{*}{ Curimatã } & $-18^{\circ} \mathrm{C}$ & 6,52 & 6,61 & 6,62 & 6,61 & 6,62 & 6,67 \\
\hline & $-36^{\circ} \mathrm{C}$ & 6,52 & 6,72 & 6,48 & 6,76 & 6,65 & 6,82 \\
\hline \multirow[t]{2}{*}{ Jaraqui } & $-18^{\circ} \mathrm{C}$ & 6,55 & 6,64 & 6,57 & 6,73 & 6,64 & 6,64 \\
\hline & $-36^{\circ} \mathrm{C}$ & 6,55 & 6,75 & 6,48 & 6,52 & 6,66 & 6,81 \\
\hline \multirow[t]{2}{*}{ Mapará } & $-18^{\circ} \mathrm{C}$ & 6,78 & 6,87 & 6,84 & 6,91 & 6,88 & 6,87 \\
\hline & $-36^{\circ} \mathrm{C}$ & 6,78 & 6,91 & 6,67 & 6,87 & 6,85 & 6,94 \\
\hline \multirow[t]{2}{*}{ Pacú } & $-18^{\circ} \mathrm{C}$ & 6,80 & 6,97 & 6,99 & 6,99 & 6,91 & 6,97 \\
\hline & $-36^{\circ} \mathrm{C}$ & 6,80 & 7,03 & 6,77 & 6,77 & 6,96 & 7,07 \\
\hline \multirow[t]{2}{*}{ Pirapitinga } & $-18^{\circ} \mathrm{C}$ & 6,50 & 6,56 & 6,56 & 6,68 & 6,44 & 6,45 \\
\hline & $-36^{\circ} \mathrm{C}$ & 6,50 & 6,71 & 6,47 & 6,71 & 6,67 & 6,78 \\
\hline \multirow[t]{2}{*}{$A+C+P(a)$} & $-18^{\circ} \mathrm{C}$ & 6,58 & 6,62 & 6,62 & 6,67 & 6,59 & 6,58 \\
\hline & $-36^{\circ} \mathrm{C}$ & 6,58 & 6,85 & 6,59 & 6,82 & 6,82 & 6,85 \\
\hline \multirow[t]{2}{*}{$J+B(b)$} & $-18^{\circ} \mathrm{C}$ & 6,61 & 6,74 & 6,66 & 6,76 & 6,80 & 6,77 \\
\hline & $-36^{\circ} \mathrm{C}$ & 6,61 & 6,78 & 6,55 & 6,67 & 6,68 & 6,77 \\
\hline
\end{tabular}

(a) "minced fish" de aracú+curimatã+pirapitinga; (b) "minced fish" de jaraqui+branquinha.
Houve tendência de aumento dos valores de TBARS ao longo da estocagem, ficando os limites mínimo e máximo entre 0,21 e 0,74mg de aldeído malônico (AM)/kg de amostra, no início do experimento, para os "minced fish" de curimatã e de J+B (jaraqui + branquinha), respectivamente. Ao final do experimento, o limite mínimo foi de $0,62 \mathrm{mg}$ de AM/kg nos "minced fish" de curimatã e de mapará estocados a $-36^{\circ} \mathrm{C}$, e o limite máximo de $3,42 \mathrm{mg}$ de $\mathrm{AM} / \mathrm{kg}$ para o de aracú, armazenado a $-18^{\circ} \mathrm{C}$ (Tabela 3). A comparação das médias ou medianas dos valores de TBARS dos "minced fish" mantidos a $-18 \pm 1^{\circ} \mathrm{C}$ e $-36 \pm 1^{\circ} \mathrm{C}$ não apresentaram diferença significante $(p>0,05)$ para a maioria dos produtos. A exceção foi para os "minced fish" de mapará $(p=0,04)$ e de pirapitinga $(p=0,03)$, sugerindo que para a maioria dos "minced fish" estudados a temperatura de $-18^{\circ} \mathrm{C}$ foi suficiente para impedir a oxidação lipídica.

TABELA 2. Valores de N-BVT (mg/100g) em "minced fish" estocados sob congelamento durante 150 dias

\begin{tabular}{|c|c|c|c|c|c|c|c|}
\hline \multirow[t]{2}{*}{ Espécies } & \multirow{2}{*}{$\begin{array}{l}\text { Temp. de } \\
\text { estocagem }\end{array}$} & \multirow[t]{2}{*}{ Início } & \multicolumn{5}{|c|}{ TEMPO DE ESTOCAGEM ( dias ) } \\
\hline & & & 30 & 60 & 90 & 120 & 150 \\
\hline \multirow[t]{2}{*}{ Aracú-comum } & $-18^{\circ} \mathrm{C}$ & 21,204 & 21,204 & 17,670 & 20,197 & 21,340 & 15,246 \\
\hline & $-36^{\circ} \mathrm{C}$ & 21,204 & 23,325 & 22,971 & 19,435 & 21,340 & 16,770 \\
\hline \multirow[t]{2}{*}{ Branquinha } & $-18^{\circ} \mathrm{C}$ & 19,084 & 20,497 & 19,791 & 18,291 & 19,435 & 16,008 \\
\hline & $-36^{\circ} \mathrm{C}$ & 19,084 & 21,558 & 19,791 & 16,386 & 18,291 & 16,770 \\
\hline \multirow[t]{2}{*}{ Curimatã } & $-18^{\circ} \mathrm{C}$ & 20,497 & 21,558 & 21,204 & 17,529 & 17,910 & 15,246 \\
\hline & $-36^{\circ} \mathrm{C}$ & 20,497 & 24,385 & 23,325 & 19,435 & 17,148 & 16,008 \\
\hline \multirow[t]{2}{*}{ Jaraqui } & $-18^{\circ} \mathrm{C}$ & 21,911 & 19,791 & 19,791 & 20,578 & 20,578 & 17,533 \\
\hline & $-36^{\circ} \mathrm{C}$ & 21,911 & 20,144 & 22,264 & 19,054 & 19,816 & 18,295 \\
\hline \multirow[t]{2}{*}{ Mapará } & $-18^{\circ} \mathrm{C}$ & 16,963 & 18,377 & 16,610 & 14,862 & 17,529 & 13,721 \\
\hline & $-36^{\circ} \mathrm{C}$ & 16,963 & 19,437 & 16,610 & 14,481 & 16,005 & 13,721 \\
\hline \multirow[t]{2}{*}{ Pacú } & $-18^{\circ} \mathrm{C}$ & 14,843 & 18,024 & 14,843 & 15,249 & 16,005 & 13,721 \\
\hline & $-36^{\circ} \mathrm{C}$ & 14,843 & 15,550 & 15,196 & 14,862 & 15,624 & 14,483 \\
\hline \multirow[t]{2}{*}{ Pirapitinga } & $-18^{\circ} \mathrm{C}$ & 19,791 & 18,377 & 20,497 & 18,291 & 18,291 & 17,533 \\
\hline & $-36^{\circ} \mathrm{C}$ & 19,791 & 22,618 & 22,618 & 18,291 & 20,578 & 17,533 \\
\hline \multirow[t]{2}{*}{$A+C+P(a)$} & $-18^{\circ} \mathrm{C}$ & 23,325 & 19,084 & 19,791 & 16,767 & 22,102 & 16,770 \\
\hline & $-36^{\circ} \mathrm{C}$ & 23,325 & 20,144 & 20,497 & 17,529 & 19,054 & 16,770 \\
\hline \multirow[t]{2}{*}{$\mathrm{J}+\mathrm{B}(\mathrm{b})$} & $-18^{\circ} \mathrm{C}$ & 19,791 & 18,377 & 20,144 & 17,910 & 20,197 & 17,533 \\
\hline & $-36^{\circ} \mathrm{C}$ & 19,791 & 20,851 & 18,730 & 18,291 & 20,197 & 18,295 \\
\hline
\end{tabular}

(A) "minced fish" de aracú+curimatã+pirapitinga;

(b) "minced fish" de jaraqui+branquinha

LANDA \& MARTINEZ [8] trabalhando com "minced fish" de peixes da fauna acompanhante da pesca de camarão, estocados em -20 a $-25^{\circ} \mathrm{C}$ durante 120 dias, mediram os valores de TBARS [micromoles de aldeído malônico $(\mathrm{AM}) / \mathrm{kg}$ ] e verificaram aumento 15,8 , no início, para 62,6 , ao final do armazenamento. Ao contrário, RODRIGUEZ \& BELLO [10] também trabalhando com o mesmo tipo de produto, congelado a $-10^{\circ} \mathrm{C}$ e a $-30^{\circ} \mathrm{C}$ durante 6 meses, encontraram diminuição progressiva nos valores de TBARS medidas em função da densidade ótica a 540nm.

JOSEPH et al [7] avaliaram a extensão da vida-útil do "minced fish" de Megalaspis cordyla ("horse mackerel"), durante estocagem a $-20^{\circ} \mathrm{C}$ por 350 dias. O grupo controle (sem antioxidantes) apresentou um aumento contínuo, tanto nos valores de peróxidos, como nos de TBARS ( $\mathrm{mg}$ de $\mathrm{AM} / \mathrm{kg}$ ), que variou de 0,55 , no início, até 8,63 , 
ao final do experimento. Esses resultados estão em concordância com o presente trabalho (Tabela 3), considerando-se os períodos de estocagem inicial e de 60 e 150 dias.

A carga microbiana inicial observada na maioria dos "minced fish" pode ser considerada relativamente alta (Tabela 4). Este fato possivelmente revela a ocorrência de deficiências no processamento, no entanto, não se verificou o crescimento de coliformes totais e foram ausentes os coliformes fecais. As contagens microbiológicas mostraram tendência geral de decréscimo ao longo do tempo de estocagem, sendo este um comportamento típico em condições de congelamento (Tabela 4). Ao final do período experimental, as contagens de bactérias mesófilas e psicrotróficas nos "minced fish" estavam abaixo dos níveis usualmente associados com a deterioração de pescado. Realmente, nenhum crescimento bacteriano deveria ser esperado nessas condições. Segundo HUSS [5], a perda da qualidade do pescado ocorre quando as contagens de bactérias aeróbias sobre a pele do peixe alcança $10^{8}-10^{9} \mathrm{UFC} / \mathrm{g}$. O ICMSF [6] estabelece que o limite máximo de bactérias aeróbias para pescado congelado deve ser de $10^{6 \mathrm{UFC}} / \mathrm{g}$.

Não foram estabelecidas correlações (teste de Pearson) significativas entre o pH (Tabela 1), N-BVT (Tabela 2), TBARS (Tabela 3) e as contagens microbiológicas (Tabela 4), que pudessem eventualmente complementar a análise dos resultados obtidos.

TABELA 3. Valores de TBARS (mg de Aldeído Malônico/kg) em "minced fish" sob congelamento durante 150 dias

\begin{tabular}{|c|c|c|c|c|c|c|c|}
\hline \multirow[t]{2}{*}{ Espécies } & \multirow{2}{*}{$\begin{array}{l}\text { Temp. de } \\
\text { estocagem }\end{array}$} & \multirow[t]{2}{*}{ Início } & \multicolumn{5}{|c|}{ TEMPO DE ESTOCAGEM ( dias ) } \\
\hline & & & 30 & 60 & 90 & 120 & 150 \\
\hline \multirow[t]{2}{*}{ Aracú-comum } & $-18^{\circ} \mathrm{C}$ & 0,237 & 0,598 & 0,746 & 1,115 & 0,764 & 3,416 \\
\hline & $-36^{\circ} \mathrm{C}$ & 0,237 & 0,437 & 0,363 & 1,179 & 0,923 & 1,262 \\
\hline \multirow[t]{2}{*}{ Branquinha } & $-18^{\circ} \mathrm{C}$ & 0,735 & 1,164 & 1,408 & 1,965 & 1,142 & 3,251 \\
\hline & $-36^{\circ} \mathrm{C}$ & 0,735 & 1,300 & 0,727 & 1,972 & 1,260 & 2,248 \\
\hline \multirow[t]{2}{*}{ Curimatã } & $-18^{\circ} \mathrm{C}$ & 0,210 & 0,323 & 0,441 & 0,769 & 1,026 & 0,869 \\
\hline & $-36^{\circ} \mathrm{C}$ & 0,210 & 0,206 & 0,165 & 0,539 & 0,489 & 0,622 \\
\hline \multirow[t]{2}{*}{ Jaraqui } & $-18^{\circ} \mathrm{C}$ & 0,550 & 0,640 & 0,656 & 0,918 & 1,133 & 1,109 \\
\hline & $-36^{\circ} \mathrm{C}$ & 0,550 & 0,484 & 0,428 & 0,913 & 0,690 & 1,453 \\
\hline \multirow[t]{2}{*}{ Mapará } & $-18^{\circ} \mathrm{C}$ & 0,223 & 0,514 & 0,677 & 0,850 & 0,652 & 0,649 \\
\hline & $-36^{\circ} \mathrm{C}$ & 0,223 & 0,351 & 0,206 & 0,429 & 0,300 & 0,603 \\
\hline \multirow[t]{2}{*}{ Pacú } & $-18^{\circ} \mathrm{C}$ & 0,520 & 0,450 & 0,861 & 1,279 & 1,256 & 1,465 \\
\hline & $-36^{\circ} \mathrm{C}$ & 0,520 & 0,510 & 0,445 & 1,482 & 0,860 & 2,966 \\
\hline \multirow[t]{2}{*}{ Pirapitinga } & $-18^{\circ} \mathrm{C}$ & 0,708 & 0,819 & 1,687 & 2,498 & 1,283 & 3,302 \\
\hline & $-36^{\circ} \mathrm{C}$ & 0,708 & 0,431 & 0,396 & 0,838 & 0,366 & 0,840 \\
\hline \multirow[t]{2}{*}{$A+C+P(a)$} & $-18^{\circ} \mathrm{C}$ & 0,438 & 0,404 & 0,600 & 1,317 & 0,624 & 1,011 \\
\hline & $-36^{\circ} \mathrm{C}$ & 0,438 & 0,497 & 0,225 & 0,789 & 0,679 & 1,081 \\
\hline \multirow[t]{2}{*}{$J+B(b)$} & $-18^{\circ} \mathrm{C}$ & 0,741 & 0,598 & 1,057 & 1,122 & 1,068 & 2,280 \\
\hline & $-36^{\circ} \mathrm{C}$ & 0,741 & 0,547 & 0,361 & 1,561 & 0,842 & 2,186 \\
\hline
\end{tabular}

(a) "minced fish" de aracú+curimatã+pirapitinga

(b) "minced fish" de jaraquitbranquinha
TABELA 4. Contagem microbiológica de "minced fish" estocados a $-18^{\circ} \mathrm{C}$ por 120 dias (a)

\begin{tabular}{|c|c|c|c|c|c|}
\hline \multicolumn{6}{|c|}{ MESÓFILOS A $35^{\circ} \mathrm{C}(\mathrm{UFC} / \mathrm{g})$} \\
\hline \multirow[t]{2}{*}{ Espécies } & \multirow[t]{2}{*}{ Início } & \multicolumn{4}{|c|}{ Tempo de Estocagem (dias) } \\
\hline & & 30 & 60 & 90 & 120 \\
\hline Aracú-comum & 6,81 & 5,15 & - & 5,18 & 5,00 \\
\hline Branquinha & 6,81 & 6,59 & 6,18 & 5,18 & 5,11 \\
\hline Curimatã & 6,81 & 6,34 & 5,23 & 5,41 & 5,41 \\
\hline Jaraqui & 6,81 & - & 4,65 & 4,88 & 4,83 \\
\hline Mapará & 5,76 & 6,81 & 6,15 & 4,72 & 4,62 \\
\hline Pacú & 6,81 & 5,18 & 5,08 & 4,91 & 4,89 \\
\hline Pirapitinga & 5,37 & 4,61 & - & 4,69 & 4,69 \\
\hline$A+C+P(b)$ & 6,81 & 6,81 & 5,23 & 4,88 & 4,83 \\
\hline$J+B(c)$ & 6,81 & 5,37 & 5,15 & 4,88 & 4,88 \\
\hline \multicolumn{6}{|c|}{ PSICROTRÓFICOS A $7^{\circ} \mathrm{C}$ (UFC/g) } \\
\hline \multirow[t]{2}{*}{ Espécies } & Início & \multicolumn{4}{|c|}{ Tempo de Estocagem (dias) } \\
\hline & & 30 & 60 & 90 & 120 \\
\hline Aracú-comum & 6,81 & 5,18 & - & 5,15 & 5,15 \\
\hline Branquinha & 6,81 & 6,41 & - & 5,08 & 5,08 \\
\hline Curimatã & 6,81 & 5,90 & 4,36 & 4,23 & 4,20 \\
\hline Jaraqui & 5,89 & - & 5,00 & 4,54 & 4,53 \\
\hline Mapará & 6,81 & 5,70 & 5,20 & 4,32 & 4,32 \\
\hline Pacú & 6,81 & 5,62 & - & 5,11 & 5,08 \\
\hline Pirapitinga & 6,53 & 5,08 & 5,18 & 5,08 & 5,08 \\
\hline$A+C+P(b)$ & 6,81 & 5,90 & 5,18 & 4,54 & 4,32 \\
\hline $\mathrm{J}+\mathrm{B}(\mathrm{c})$ & 6,81 & 5,18 & 5,15 & 4,72 & 4,72 \\
\hline
\end{tabular}

(a) Valores em escala logarítmica;

(b) "minced fish" de aracú+curimatã+pirapitinga;

(c) "minced fish" de jaraqui+branquinha.

\section{4 - CONCLUSÕES}

Os resultados alcançados permitem concluir que as diferentes espécies estudadas possuem características promissoras do ponto de vista tecnológico. Os "minced fish" obtidos de espécies e misturas de espécies de peixes amazônicas mantiveram-se estáveis sob os aspectos químico e microbiológico durante 150 dias a $-18 \pm 1^{\circ} \mathrm{C}$ e $-36 \pm 1^{\circ} \mathrm{C}$, portanto em condições de consumo.

\section{5 - REFERÊNCIAS BIBLIOGRÁFICAS}

[1] BRASIL. Ministério da Agricultura. Laboratório Nacional de Referência Animal. Métodos analíticos oficiais para controle de produtos de origem animal e seus ingredientes. 1. Métodos microbiológicos. Brasília: LANARA, 1981. 112p.

[2] FAO/WHO Draft Revised Standard for Quick Frozen Blocks of Fish Fillets, Minced Fish Flesh and Mixtures of Fillets and Minced Fish Flesh (Appendix IV). In: Report of the 21 $1^{\text {st }}$ Session of the Codex Committee on Fish and Fishery Products. Rome: Codex Alimentarius Commission, 1994. p. 47-57. 
[3] FEDERAÇÃO DOS PESCADORES DO AMAZONAS E RORAIMA. Perfil econômico do setor pesqueiro do Estado do Amazonas. Manaus, 1998. 10p. (Relatório).

[4] GUTIÉRREZ, M., BELLO, R.A. Estudio sobre la factibilidad de elaboración de productos congelados a partir de pulpa de pescado proveniente de especies subutilizadas. México (DF): Secretaria de Pesca,1991. p. 21-34. (Revista Tecnología de los Alimentos, n. 4).

[5] HUSS, H.H. Fresh fish: quality and quality changes. Rome, FAO, DANIDA, 1988. p. 43-45.

[6] INTERNATIONAL COMMISSION ON MICROBIOLOGICAL SPECIFICATIONS FOR FOODS. Microrganismos de los alimentos. Técnicas de análisis microbiológicos. Zaragoza: Ed. Acribia, 1983. v. 1, 533p.

[7] JOSEPH, J., GEORGE, C., PERIGREEN, P.A. Effect of spices on improving the stability of frozen stored fish mince. Fisheries Technology, Kochi, v. 29, p. 30-34, 1992.

[8] LANDA, L.F.B., MARTINEZ, R.F. Informe técnico de avances sobre la normalización de la pulpa de pescado obtenida de la fauna de acompañamiento del camaron. México (DF): Secretaria de Pesca, 1991. p. 60-80. (Revista Tecnologia de los Alimentos, n. 4).

[9] LOVE, R.M. Biochemical dynamics and the quality of fresh and frozen fish. In: HALL, G.M. ed., Fish Processing Technology.Glasgow: Blackie Academic \& Professional, 1992. p.1-31.
[10] RODRIGUEZ G., L., BELLO, R.A. Elaboracion de bloques congelados de pulpa de pescado y su evaluacion durante el almacenamiento. Archivos Latinoamericanos de Nutrición, Caracas, v. 37, n. 2, p. 351-363, 1987.

[11] SÃO PAULO. Secretária da Saúde. Código Sanitário. São Paulo, 1991. NTA-9, p. 167-168.

[12] SÃO PAULO. Secretária da Saúde. Normas analíticas do Instituto Adolfo Lutz. I. Métodos químicos e físicos para análise de alimentos. 3. ed. São Paulo, 1985. 533p.

[13] WOOTLON, M., CHUAH, S.H. The use of sea mullet (Mugil cephalus) in the production of cold marinades. Food Technology in Australia, Sidney, v. 33, n. 8, p. 392-397, 1981.

[14] WYNCKE, W.. Direct determination of the thiobarbituric acid value in trichloroacetic extracts of fish as a measure of oxidative rancidity. Fette Seifen Anstrichmiltel, Leinfelden, n. 12, p. 1084-1087, 1970.

[15] ZAR, J.H. Biostatistical analysis. 3. ed. London: PrenticeHall, 1994. 606p.

\section{6 - AGRADECIMENTOS}

Ao Dr. Paulo de Tarso Falcão, do INPA/CPTA

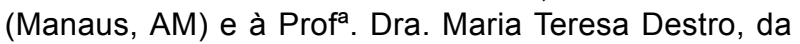
USP/FCF/FBA (São Paulo, SP), pelo auxílio na realização das análises microbiológicas. 